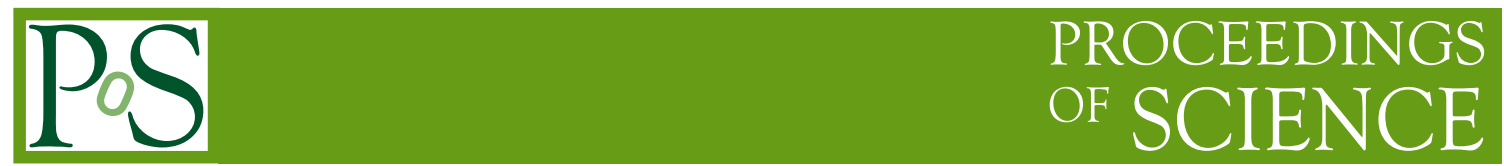

\title{
Recent BESIII results in charmonium physics
}

\section{Yaqian Wang ${ }^{* \dagger}$}

Institute of Nuclear Physics, Mainz University

E-mail: whyaqmegmail.com

Based on the world largest $\psi^{\prime}$ data sample collected with the BESIII experiment at the BEPCII collider, we performed measurements on $h_{c}, \eta_{c}$, and $\eta_{c}$ '. The results include the masses, widths, and production rates of these resonances.

Xth Quark Confinement and the Hadron Spectrum,

October 8-12, 2012

TUM Campus Garching, Munich, Germany

* Speaker.

$\dagger$ On behalf of the BESIII Collaboration. 


\section{Introduction}

BEPCII/BESIII [1] is an upgrade facility from the previous BEPC/BESII experiment. The BESIII spectrometer consists of a main drift chamber with momentum resolution $0.5 \%$ at $1 \mathrm{GeV} / c$, an electromagnetic calorimeter with energy resolution $2.5 \%$ at $1.0 \mathrm{GeV}$, a Time-Of-Flight counter, a superconducting magnet with a magnetic strength of $1 \mathrm{~T}$, and a muon chamber system made of resistive plate chambers.

Based on $106 \times 10^{6}$ [2] $\psi^{\prime}$ events taken in 2009, charmonium spectroscopy is widely studied.

\section{Measurements of $h_{c}$ with $\psi^{\prime} \rightarrow \pi^{0} h_{c}$}

As the P-wave spin singlet, $h_{c}$ is first observed by E835 experiment [3] with scanning antiproton energy. Later, CLEO Collaboration observed the $h_{c}$ in the cascade process $\psi^{\prime} \rightarrow \pi^{0} h_{c}, h_{c} \rightarrow \gamma \eta_{c}$ with both inclusive and exclusive measurements $[4,5]$. There are theoretical predictions [6] for the production and decays of $h_{c}$, which can be tested with the largest $\psi^{\prime}$ data sample at BESIII. Both E1-tagged and E1-untagged method in $h_{c} \rightarrow \gamma \eta_{c}$ are performed at BESIII to measure the branching fraction of $\psi^{\prime} \rightarrow \pi^{0} h_{c}$ [7]. The results are shown in Fig. 1. The mass and width of $h_{c}$ determined

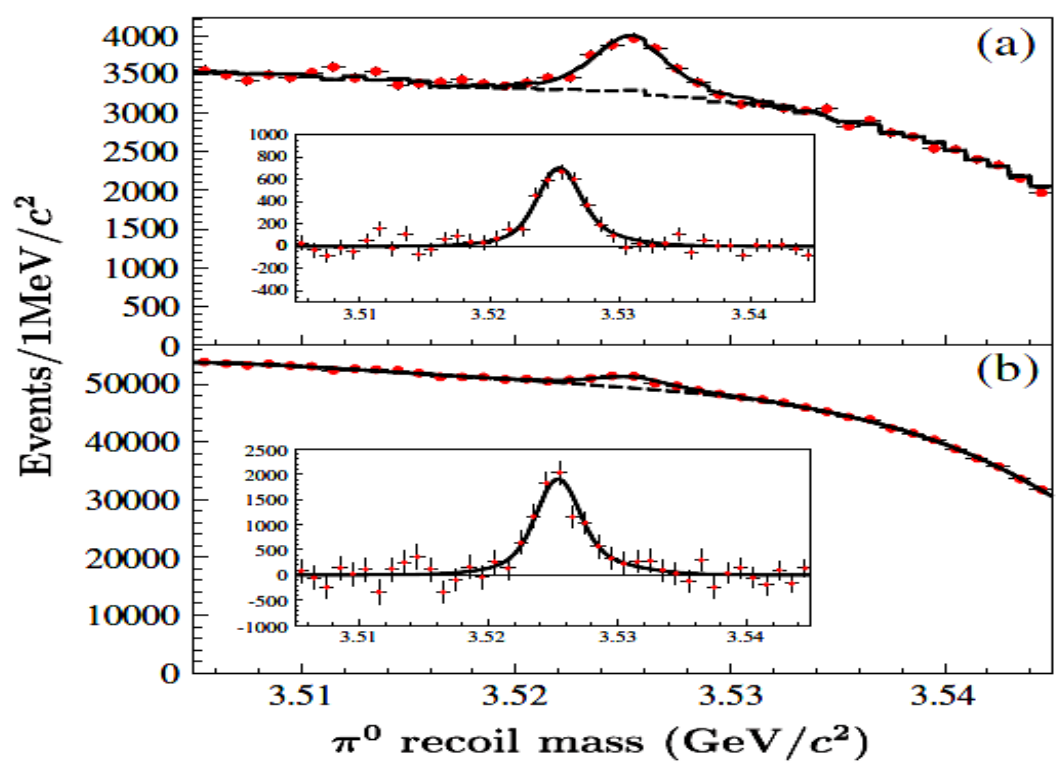

Figure 1: The recoiling mass of the tagged $\pi^{0}$ in $\psi^{\prime} \rightarrow \pi^{0} h_{c}$ : (a) for the E1 tagged method, (b) for the E1 untagged method.

with the E1 tagged method are $M_{h_{c}}=3525.40 \pm 0.13 \pm 0.18 \mathrm{MeV} / c^{2}$ and $\Gamma_{h_{c}}=0.73 \pm 0.45 \pm 0.28$ $\mathrm{MeV}$. The mass split $\Delta M_{h f} \equiv\left\langle M\left(1^{3} P\right)\right\rangle-M\left(1^{1} P_{1}\right)=-0.10 \pm 0.13 \pm 0.18 \mathrm{MeV} / c^{2}$, which is consistent with zero predicted by the potential model. The branching fractions are determined to be $\mathscr{B}\left(\psi^{\prime} \rightarrow \pi^{0} h_{c}\right)=(8.3 \pm 1.3 \pm 1.0) \times 10^{-4}$ and $\mathscr{B}\left(h_{c} \rightarrow \gamma \eta_{c}\right)=(54.3 \pm 6.7 \pm 5.2) \%$, which also agree with the theoretical predictions. Exclusive measurements are also studied with sixteen $\eta_{c}$ decay channels, and the simultaneous fit is shown in Fig. 2. Results from the exclusive measurement are: $M_{h_{c}}=3525.31 \pm 0.11 \pm 0.14 \mathrm{MeV} / c^{2}$, and $\Gamma_{h_{c}}=0.70 \pm 0.28 \pm 0.22 \mathrm{MeV}$. They are consistent with the previous measurement at CLEO [5]. 

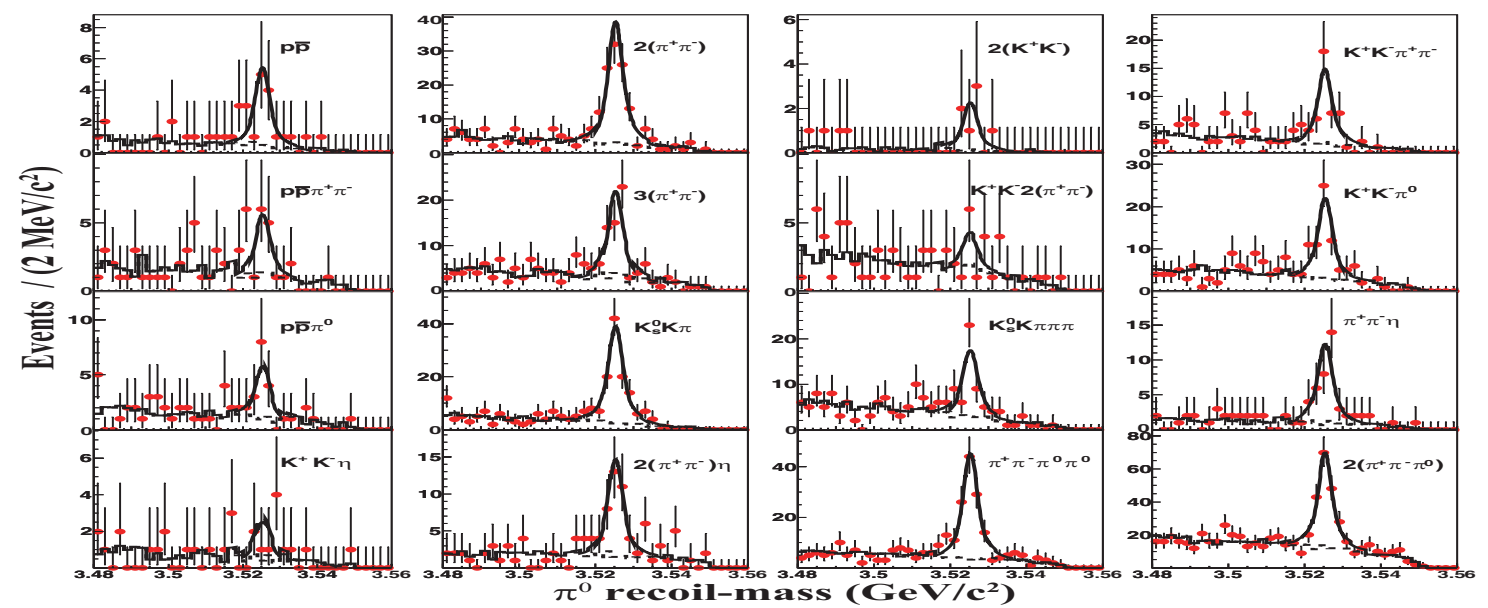

Figure 2: Simultaneous fit to 16 exclusive decay modes.

\section{Measurements of the mass and width of the $\eta_{c}$ using $\psi^{\prime} \rightarrow \gamma \eta_{c}$}

Properties of $\eta_{c}$ are not well understood although it has been observed for many years. There are obvious discrepancies between results from different experiments [8]. An obvious distortion in the line shape of $\eta_{c}$ is reported by CLEO [9], but similar effect is not observed in $\psi^{\prime} \rightarrow \pi^{0} h_{c}$, $h_{c} \rightarrow \gamma \eta_{c}$ at BESIII.

Measurement of $\eta_{c}$ at BESIII is performed with six decay channels, including $K_{S}^{0} K \pi, K^{+} K^{-} \pi^{0}$, $\pi^{+} \pi^{-} \eta, K_{S}^{0} K 3 \pi, K^{+} K^{-} \pi^{+} \pi^{-} \pi^{0}$ and $3\left(\pi^{+} \pi^{-}\right)$[10]. A simultaneous fit with unique $\eta_{c}$ mass and width is performed on the $\eta_{c}$ mass spectra, where a full interference between $\eta_{c}$ and non-resonant $\psi^{\prime}$ radiative decays is considered and the quantum number of the non- $\eta_{c}$ components are assumed to be $0^{-+}$. The corresponding relative phases in different decay modes are found to be consistent within $3 \sigma$, which are constrained to the same value in the final fit. The simultaneous fit is shown in Fig. 3. The obtained results are $M_{\eta_{c}}=2984.3 \pm 0.6 \pm 0.6 \mathrm{MeV} / c^{2}, \Gamma_{\eta_{c}}=32.0 \pm 1.2 \pm 1.0 \mathrm{MeV}$, $\phi_{1}=2.40 \pm 0.07 \pm 0.08 \mathrm{rad}$ (constructive), and $\phi_{2}=4.19 \pm 0.03 \pm 0.09 \mathrm{rad}$ (destructive). The BESIII results are consistent with that from two-photon production $[11,12,13]$. The precision of the measured mass and width are improved.

\section{First observation of the M1 transition $\psi^{\prime} \rightarrow \gamma \eta_{c}^{\prime}$}

$\eta_{c}^{\prime}$ is first observed by Belle Collaboration [14] in $B \rightarrow K K_{S} K^{-} \pi^{+}$, which is also expected in the radiative transition of $\psi^{\prime}$. According to the potential model, the branching fraction is predicted to be $\mathscr{B}\left(\psi^{\prime} \rightarrow \gamma \eta_{c}^{\prime}\right)=(0.1-6.2) \times 10^{-4}[15]$.

BESIII performed a searching for the $\eta_{c}^{\prime}$ in several decay modes, and signal is only observed in the $K \bar{K} \pi$ [16] final state. Figure 4 shows the fit result to the mass spectrum of $K \bar{K} \pi$. With a simultaneous fit we obtain the mass and width of $\eta_{c}^{\prime}: M_{\eta_{c}^{\prime}}=3637.6 \pm 2.9 \pm 1.6 \mathrm{MeV} / c^{2}$, and $\Gamma_{\eta_{c}^{\prime}}=16.9 \pm 6.4 \pm 4.8 \mathrm{MeV}$. The product branching fraction is determined to be $\mathscr{B}\left(\psi^{\prime} \rightarrow \gamma \eta_{c}^{\prime}\right) \times$ $\mathscr{B}\left(\eta_{c}^{\prime} \rightarrow K \bar{K} \pi\right)=(1.30 \pm 0.20 \pm 0.30) \times 10^{-5}$. Using the previous measurement $\mathscr{B}\left(\eta_{c}^{\prime} \rightarrow K \bar{K} \pi\right)=$ 

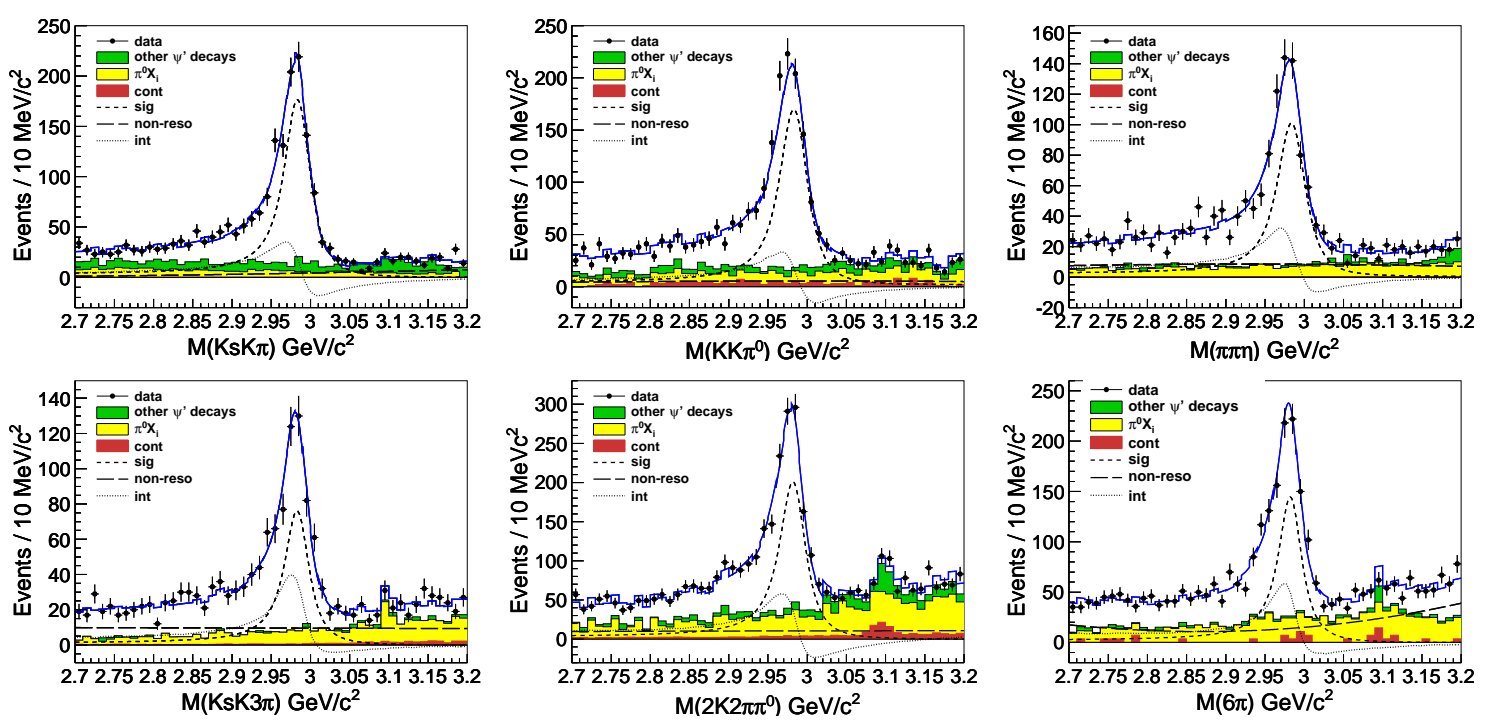

Figure 3: The simultaneous fit of six decay channels.
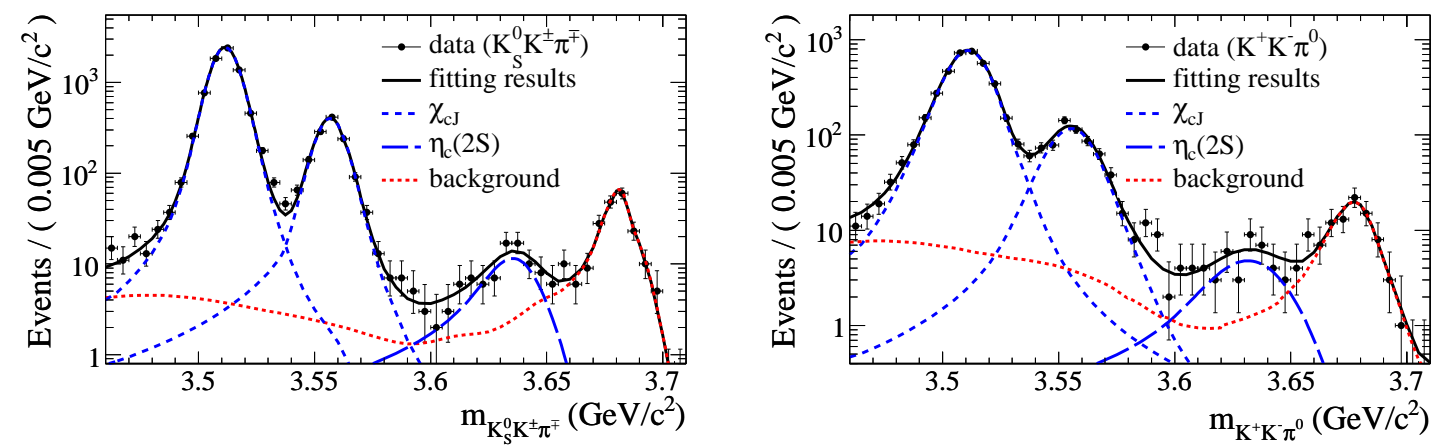

Figure 4: The simultaneous fit to the $K \bar{K} \pi$ mass spectrum.

$(1.9 \pm 0.4 \pm 1.1) \%$ from BaBar [17], the M1 transition rate is determined to be $\mathscr{B}\left(\psi^{\prime} \rightarrow \gamma \eta_{c}^{\prime}\right)=$ $(6.8 \pm 1.1 \pm 4.5) \times 10^{-4}$.

Searching for $\eta_{c}^{\prime}$ is also performed with the $V V$ final states, including $\rho^{0} \rho^{0}, K^{* 0} \bar{K}^{* 0}$, and $\phi \phi$. The intermediate charmed meson loops can be taken as a mechanism to evade the helicity selection rule, which provides possible explanation for the long standing " $\rho \pi$ " puzzle. Considering this effect, the production of $\eta_{c}^{\prime} \rightarrow V V$ is expected to be quite high. Based on $106 \mathrm{M} \psi^{\prime}$ data, no significant $\eta_{c}^{\prime}$ signal is found in the three final states we studied, and the branching fraction upper limits are determined to be $\mathscr{B}\left(\eta_{c}^{\prime} \rightarrow \rho^{0} \rho^{0}\right)<3.1 \times 10^{-3}, \mathscr{B}\left(\eta_{c}^{\prime} \rightarrow K^{* 0} \bar{K}^{* 0}\right)<5.4 \times 10^{-3}$, and $\mathscr{B}\left(\eta_{c}^{\prime} \rightarrow \phi \phi\right)<2.0 \times 10^{-3}$ [18], which are lower than the theoretical predictions [19]. 


\section{Summary}

With the $106 \times 10^{6} \psi^{\prime}$ data collected by BESIII, the following results about Charmonium spectroscopy are obtained: the properties of $h_{c}$ are measured with both inclusive and exclusive methods; the properties of $\eta_{c}$ are precisely measured using the radiative decays of $\psi^{\prime}$, where the interference between $\eta_{c}$ decays and non-resonant decays is taken into account; the M1 transition $\psi^{\prime} \rightarrow \gamma \eta_{c}^{\prime}$ is first observed with the $K \bar{K} \pi$ final state.

\section{Acknowledgements}

We thank the BEPCII group for excellent operation of the accelerator, the IHEP computer group for valuable computing and network support, and all the colleagues contributing on the physical measurements.

\section{References}

[1] M. Ablikim et al. (BESIII Collaboration), Nucl. Instrum. Methods Phys. Res., Sect. A 614, 345 (2010).

[2] M. Ablikim et al. (BESIII Collaboration), arXiv:1209.6199.

[3] M. Andreotti et al. (E-835 Collaboration), Phys. Rev. D 72, 032001 (2005).

[4] S. Dobbs et al. (CLEO Collaboration), Phys. Rev. Lett. 101, 182003 (2008).

[5] G.S. Adams et al. (CLEO Collaboration), Phys. Rev. D 80, 051106 (2009).

[6] Y.P. Kuang, Phys. Rev. D 65, 094024 (2002); S. Godfrey and J.L. Rosner, Phys. Rev. D 66, 014012 (2002).

[7] M. Ablikim et al. (BESIII Collaboration), Phys. Rev. Lett. 104, 132002 (2010).

[8] K. Nakamura et al., Journal of Physics G 37, 075021 (2010).

[9] R.E. Mitchell et al. (CLEO Collaboration), Phys. Rev. Lett. 102, 011801 (2009).

[10] M. Ablikim et al. (BESIII Collaboration), Phys. Rev. Lett. 108, 222002 (2012).

[11] D. M. Asner et al. (CLEO Collaboration), Phys. Rev. Lett. 92, 142001 (2004).

[12] B. Aubert et al. (BABAR Collaboration), Phys. Rev. Lett. 92, 142002 (2004).

[13] S. Uehara et al. (Belle Collaboration), Eur. Phys. J. C 53, 1 (2008).

[14] S.K. Choi et al. (Belle Collaboration), Phys. Rev. Lett. 89, 102001 (2002).

[15] K. Gao, PhD thesis, arXiv: 0909.2812.

[16] M. Ablikim et al. (BESIII Collaboration), Phys. Rev. Lett. 109, 042003 (2012).

[17] B. Aubert et al. (BABAR Collaboration), Phys. Rev. D 78, 012006 (2008).

[18] M. Ablikim et al. (BESIII Collaboration), Phys. Rev. D 84, 091102 (2011).

[19] Q. Wang, X. H. Liu and Q. Zhao, arXiv:1010.1343. 